\title{
Automated Screening for Oxidative or Methylation-Induced DNA Damage in Human Cells
}

\author{
Matthias Mack, Katharina Schweinlin, Nicola Mirsberger, Tabea Zubel and Alexander Bürkle \\ Molecular Toxicology Group, Department of Biology, University of Konstanz, Konstanz, Germany
}

\begin{abstract}
The assessment of genotoxicity upon exposure to chemical and environmental agents plays an important role in basic research as well as in pharmaceutical, chemical, cosmetic and food industry. Low sensitivity and lack of inter-laboratory comparability are considered problematic issues in genotoxicity testing. Moreover, commonly used mutagenicity assays lack information about early and specific genotoxic events.

Previously, we developed an automated version of the "Fluorimetric detection of Alkaline DNA Unwinding" (FADU) assay as a high-throughput screening method for the detection of DNA strand breaks in living cells. Here, we report an enzyme-modified version of the cell-based FADU assay (emFADU) for the determination of oxidative and methylation lesions in cellular DNA. Our method is based on the use of formamidopyrimidine DNA glycosylase or human alkyladenine DNA glycosylase for the detection of chemically-induced nucleobase modifications in lysates of immortalized cell lines, growing in suspension or as adherent cells, and in peripheral blood mononuclear cells. We could show that upon treatment with sub-cytotoxic doses of known genotoxins, oxidative and methylation lesions are readily detectable.

This fast, inexpensive, and convenient method could be useful as a high-content screening approach for the sensitive and specific assessment of genotoxicity in human cells. Thus, when implemented in the early compound development in an industrial setting, the emFADU assay could help reduce the number of animals used for toxicity testing. Furthermore, as we established the method for different cell types, this new assay may provide an opportunity for population studies and/or mechanistic research into DNA repair pathways.
\end{abstract}

\section{Introduction}

Every day, a human cell must cope with a variety of DNA insults, including DNA strand breaks (SB), apurinic/apyrimidinic (AP) sites and nucleobase lesions (Lindahl, 1993). Therefore, highly efficient DNA repair mechanisms have evolved to safeguard the integrity of the human genome. Unrepaired DNA damage could lead to cell death, resulting in accelerated aging, or mutations, increasing the risk of cancer development (Bernstein et al., 2002; Hoeijmakers, 2009). Besides endogenous DNA damage, our genome is constantly challenged by a variety of exogenous genotoxic sources, e.g., tobacco smoke, air pollution, processed meat, and conceivably unknown chemicals in the environment, highlighting the necessity of genotoxicity testing (Bouvard et al., 2015; Ciccia and Elledge, 2010; Cross and Sinha, 2004). Furthermore, ongoing product development in pharmaceutical, chemical, cosmetic and food industry requires sensitive, robust, and cost-effective meth- ods for risk assessment and classification of chemicals. When establishing new test methods, it is imperative to implement the $3 \mathrm{R}$ principle where possible. Ideally such tests should be animal-free and should rely on human-relevant biological systems (Beken et al., 2016; Knudsen et al., 2019).

The comet assay is the gold standard in many laboratories for genotoxicity measurements. With its high sensitivity and broad scope of application, it is an attractive technique for analyzing DNA damage induction and repair in a broad variety of cells. Nevertheless, a problematic issue is the lack of inter-laboratory comparability (Ersson et al., 2013). Work on standardization of the comet assay protocol has decreased the inter-experimental and inter-laboratory variability of the assay (Cassano et al., 2020), but also shown its drawbacks (Brunborg et al., 2018; Enciso et al., 2018). The CometChip ${ }^{\circledR}$ platform provides a high-throughput DNA damage analysis in a 96-well format, but no automated version has been described so far (Sykora et al., 2018). Additionally,
Received January 22, 2020; Accepted July 8, 2020;

Epub July 11, 2020; (C) The Authors, 2020.

ALTEX 38(1), 063-072. doi:10.14573/altex.2001221

Correspondence: Alexander Bürkle, MD

Molecular Toxicology Group

Department of Biology

University of Konstanz

Universitätsstr. 10, 78464 Konstanz, Germany

(alexander.buerkle@uni-konstanz.de)
This is an Open Access article distributed under the terms of the Creative Commons Attribution 4.0 International license (http://creativecommons.org/licenses/by/4.0/) which permits unrestricted use, distribution and reproduction in any medium, provided the original work is appropriately cited. 
costs per run remain high ( $>200 €$ per chip), limiting its use especially for big sample sizes.

As an alternative to the comet assay, we have developed an automated version of the "Fluorimetric detection of Alkaline DNA Unwinding" (FADU) assay (Moreno-Villanueva et al., 2009, 2011). First described by Birnboim and Jevcak (1981), the method uses a highly alkaline $\mathrm{pH}$ for unwinding of genomic DNA in a cell lysate, starting at chromosome ends and sites of replication. DNA SBs serve as additional starting points for DNA unwinding. Therefore, increased numbers of SBs lead to increased unwinding, which can be quantified by measuring the amount of DNA remaining double stranded during the alkaline unwinding phase of the assay, as detected via SYBR ${ }^{\text {TM }}$ Green I staining. Based on the use of a liquid handling device and a 96-well format, we have been able to reduce operator bias and increase sample throughput. In a previous study, we could show that the FADU principle can be used to detect chemically induced formamidopyrimidine DNA glycosylase (Fpg)-sensitive sites in purified plasmid DNA (Müller et al., 2013). The glycosylase recognizes and excises oxidative DNA lesions, like 8-oxo-2'-deoxyguanosine (8-oxodG), leaving an apurinic/apyrimidinic (AP) site that can be processed by Fpg's endonuclease function, resulting in a DNA SB (O'Connor and Laval, 1989; Tchou et al., 1991). In the present work, we could also show that the FADU can be used to detect methyl methanesulfonate (MMS) induced methylation lesions in purified plasmid DNA by using the human alkyladenine DNA glycosylase (hAAG) in combination with AP endonuclease 1 (APE1) (Fig. S1 ${ }^{1}$ ).

Already more than 20 years ago, glycosylases were used to assess DNA damage in human cells. This was implemented in alkaline elution and alkaline unwinding to detect oxidative DNA damage (Hartwig et al., 1996; Pflaum et al., 1997). However, the methods had to be performed manually and are not suitable for analyzing larger numbers of samples at high throughput. Here, we describe a novel method for the detection of genotoxicity in human cells. This was implemented by modifying the automated FADU assay for the detection of glycosylase-specific DNA lesions to assess the amount of oxidative DNA lesions as well as methylation lesions. We found that subcytotoxic doses of hydrogen peroxide $\left(\mathrm{H}_{2} \mathrm{O}_{2}\right)$, potassium bromate $\left(\mathrm{KBrO}_{3}\right)$ and MMS lead to a significant increase in nucleobase modifications, thus demonstrating the proof-of-principle to increase the sensitivity of genotoxicity testing with our new technique. Regarding workload, assay duration and cost efficiency, we show that the emFADU is an attractive alternative to the CometChip ${ }^{\circledR}$ platform.

\section{Materials and methods}

\subsection{Cell culture}

THP-1 cells were cultivated in RPMI 1640 medium supplemented with $10 \%$ fetal calf serum (FCS; Biochrome) and $1 \%$ penicillin-streptomycin (PenStrep; Gibco). Cells were kept at $37^{\circ} \mathrm{C}$ in a humidified atmosphere with $5 \% \mathrm{CO}_{2}$. Cells were cryopreserved to create stock cultures. Cells were routinely tested for mycoplasma contamination (once per year and prior to every master stock preparation).

Venous blood was obtained from apparently healthy human donors aged 23-29 (two thirds women, one third men). Selection of volunteers was carried out in accordance with the Declaration of Helsinki, and ethical approval was obtained from the Ethics Committee of the University of Konstanz. Signed informed consent was obtained from each subject. Peripheral blood mononuclear cells (PBMCs) were isolated using Biocoll density centrifugation. Briefly, $10 \mathrm{~mL}$ whole blood was drawn with citrate as anti-coagulant. The blood was diluted with an equal volume of phosphate buffered saline (PBS; Biochrome), layered on top of $15 \mathrm{~mL}$ Biocoll separation solution (Biochrome), and centrifuged at $900 \mathrm{~g}$ for $15 \mathrm{~min}$ at RT in a swing-out rotor without break. PBMCs were harvested from the gradient and washed in PBS by centrifugation at $300 \mathrm{~g}$ for $10 \mathrm{~min}$ at $4^{\circ} \mathrm{C}$. After washing, PBMCs were resuspended in pre-warmed RPMI 1640 medium supplemented with $10 \%$ FCS and $1 \%$ PenStrep.

HeLa Kyoto cells were cultivated in DMEM (Dulbecco's Modified Eagle's Medium: Gibco), supplemented with $10 \%$ FCS and $1 \%$ PenStrep. Cells were kept at $37^{\circ} \mathrm{C}$ in a humidified atmosphere with $5 \% \mathrm{CO}_{2}$. Cells were routinely tested for mycoplasma contamination (once per year and prior to every master stock preparation).

Cultivation of cells was established and conducted with the use of FCS. For future studies we aim to investigate alternatives to the use of FCS in order to replace the use of animal-derived products.

\subsection{Genotoxic treatment}

For X-irradiation, $300 \mu \mathrm{L}$ cell suspension (THP-1: $6.7 \times 10^{5}$ cells/ $\mathrm{mL}$; PBMCs: $13.4 \times 10^{5}$ ) were irradiated in reaction tubes on ice using an X-RAD 225 - Precision X-ray irradiator. Cells were washed twice with $900 \mu \mathrm{L}$ ice-cold PBS and resuspended in 300 $\mu \mathrm{L}$ PBS containing $300 \mu \mathrm{M}$ deferoxamine mesylate salt (DFA; CAS: 138-14-7, Sigma-Aldrich) and $300 \mu \mathrm{M} 4$ hydroxy-TEMPO (TEMPOL; CAS: 2226-96-2, Sigma-Aldrich).

For genotoxic treatment of THP-1 cells and PBMCs, $500 \mu \mathrm{L}$ cell suspension (THP-1: $6.7 \times 10^{5}$ cells $/ \mathrm{mL}$; PBMCs: $13.4 \times 10^{5}$ ) was transferred to a 24-well plate for each sample. The genotoxin was pre-diluted in growth medium, and cells were treated with various concentrations at $37^{\circ} \mathrm{C}$ in a humidified atmosphere with $5 \%$ $\mathrm{CO}_{2}$. The time of incubation for $\mathrm{H}_{2} \mathrm{O}_{2}$ (CAS: 7722-84-1, Sigma-Aldrich) was set to $30 \mathrm{~min}$, and to $120 \mathrm{~min}$ for $\mathrm{KBrO}_{3}$ (CAS: 775801-2, Sigma-Aldrich) or MMS (CAS: 66-27-3, Sigma-Aldrich). After treatment, $300 \mu \mathrm{L}$ cell suspension was transferred to a reaction tube and used for cell harvest $\left(300 \mathrm{~g}, 4^{\circ} \mathrm{C}, 10 \mathrm{~min}\right)$, washed twice with ice-cold $900 \mu \mathrm{L}$ PBS, and resuspended in $300 \mu \mathrm{L}$ PBS containing $300 \mu \mathrm{M}$ DFA and $300 \mu \mathrm{M}$ TEMPOL.

For genotoxic treatment of HeLa cells, $15 \mu \mathrm{L}$ cell suspension $\left(3.3 \times 10^{5}\right.$ cells $\left./ \mathrm{mL}\right)$ was seeded per well in a Falcon ${ }^{\circledR} 96$-well Clear Round Bottom TC-treated Cell Culture Microplate. After $2 \mathrm{~h}$, $85 \mu \mathrm{L}$ culture medium was added to avoid desiccation. The next day, the genotoxin was pre-diluted in growth medium and cells

1 doi:10.14573/altex.2001221s 
were treated with various concentrations at $37^{\circ} \mathrm{C}$ in a humidified atmosphere with $5 \% \mathrm{CO}_{2}$. For each condition, 12 wells were treated equally, i.e., 3 replicates each for T values (= total DNA), $\mathrm{P}$ values (= physiological DNA SBs), F values (= Fpg-sensitive sites), and hA values (= hAAG/APE1-sensitive sites). The time of exposure to $\mathrm{KBrO}_{3}$ and $\mathrm{MMS}$ was set to $120 \mathrm{~min}$. After treatment, all wells were washed twice with $200 \mu \mathrm{L}$ ice-cold PBS.

\subsection{Detection of DNA strand breaks and glycosylase- specific DNA lesions}

For the detection of DNA base lesions (oxidative and methylation lesions) in cells, the automated FADU assay (Moreno-Villanueva et al., 2009) was modified in order to increase the sensitivity and extend the scope of application. Besides the $\mathrm{T}$ value, representing the total amount of DNA, and the P value, reflecting the extent of DNA strand breaks, two additional parameters for enzyme-specific DNA lesions were established. Due to incubation with lesion-specific glycosylases, these values reflect the numbers of directly induced DNA strand breaks plus enzyme-induced strand breaks. In particular, the F value reflects the extent of DNA strand breaks and Fpg-sensitive sites. The hA value reflects the extent of DNA strand breaks and hAAG/APE1-sensitive sites. All automated steps of the emFADU, performed by a TECAN Genesis RSP 100 liquid handling device, are illustrated in Figure 1A.

\section{Cell transfer and lysis}

The samples of suspension cells had to be transferred into 96-well plates. For each sample of THP-1 cells or PBMCs, 12 wells of a 96-well plate master block with V-shaped bottom (Greiner-Bio One GmbH, Frickenhausen, Germany), which was cut horizontally to hold $1 \mathrm{~mL}$ volume, were filled with $15 \mu \mathrm{L}$ cell suspension ( 3 replicates each for $\mathrm{T}=$ total $\mathrm{DNA}, \mathrm{P}=$ physiological DNA SBs, $\mathrm{F}$ value $=$ Fpg-sensitive sites, hA $=$ hAAG/APE1-sensitive sites). $15 \mu \mathrm{L}$ lysis buffer (400 mM guanidine thiocyanate; CAS: 593-84-0, Sigma-Aldrich; 30 mM EDTA, Sigma-Aldrich; 0.2\% N-laurylsarcosine; CAS: 137-16-6, Sigma-Aldrich; $300 \mu \mathrm{M}$ DFA and $300 \mu \mathrm{M}$ TEMPOL, adjusted to $\mathrm{pH} 12$ with $\mathrm{NaOH}, \mathrm{CAS}$ : 1310-73-2, Sigma-Aldrich) was added. Cell suspension, lysis buffer and the well plate were all kept at $4^{\circ} \mathrm{C}$.

HeLa cells were already seeded, treated, and washed in a 96-well plate. The supernatant was removed and 7.5 $\mu \mathrm{L}$ PBS (supplemented with $300 \mu \mathrm{M}$ DFA and $300 \mu \mathrm{M}$ TEMPOL) was added. 7.5 $\mu \mathrm{L}$ lysis buffer was added. Cell suspension, lysis buffer, and the well plate were all kept at $4^{\circ} \mathrm{C}$. For the following steps, all volumes described for suspension cells were reduced to $50 \%$. 12 equally treated wells were divided into 3 replicates for each value ( $\mathrm{T}, \mathrm{P}, \mathrm{F}$ and $\mathrm{hA}$ ).

\section{Dilution and enzyme incubation}

After 12 min of lysis at $4^{\circ} \mathrm{C}, 270 \mu \mathrm{L}$ dilution buffer $(2 \mathrm{mM}$ HEPES; CAS: 1365-25-9, Sigma-Aldrich; $300 \mu \mathrm{M}$ DFA and $300 \mu \mathrm{M}$ TEMPOL) was added on top of the lysate of each well at a dispensing rate of $10 \mu \mathrm{L} / \mathrm{s}$. For data points with enzyme addition, the dilution buffer was supplemented with lesion-specific enzymes: 0.2 U/mL Fpg (M0240S, New England BioLabs), 0.8 U/mL hAAG (M0313S, New England BioLabs) + $0.05 \mathrm{U} / \mathrm{mL}$
APE1 (M9282S, New England BioLabs). After 3 min diffusion, the 96 -well plate was transferred to $37^{\circ} \mathrm{C}$ for a $1-\mathrm{h}$ incubation.

\section{Alkaline DNA unwinding and neutralization}

The plate was transferred back to the cooling device and cooled down to $4^{\circ} \mathrm{C}$ for $3 \mathrm{~min}$. First, the replicates of the T value received $150 \mu \mathrm{L}$ neutralization solution (14 mM $\beta$-mercaptoethanol; CAS: 60-24-2, Merck; 1 M D(+)-glucose monohydrate; CAS: 1443143-7, Sigma-Aldrich). $50 \mu \mathrm{L}$ alkaline solution (160 mM guanidine thiocyanate, Sigma-Aldric; $12 \mathrm{mM}$ EDTA; 0.08\% N-lauroylsarcosyl; $300 \mathrm{mM} \mathrm{NaOH}$ ) was added to all wells on top of the solution at a dispensing rate of $10 \mu \mathrm{L} / \mathrm{s}$. After 5 min diffusion, the temperature was set to $30^{\circ} \mathrm{C}$ for $1 \mathrm{~h}$ to allow DNA unwinding. Then, the temperature was set to $20^{\circ} \mathrm{C}$ to reach RT within $30 \mathrm{~min}$. Thereafter, wells for P, F and hA values were neutralized with 150 $\mu \mathrm{L}$ neutralization solution.

\section{SYBR ${ }^{\mathrm{TM}}$ Green staining and fluorescence read-out}

For the determination of double-stranded DNA, $50 \mu \mathrm{L}$ SybrGreen $^{\mathrm{TM}}$ I (S7563, Invitrogen) solution (1:2,700 SYBR ${ }^{\mathrm{TM}}$ Green in $\mathrm{H}_{2} \mathrm{O}$ ) was added to each well and the content was mixed 6 times. Fluorescence intensity (excitation: $485 \mathrm{~nm}$, emission: 535 $\mathrm{nm}$ ) was measured by using a TECAN infinite ${ }^{\mathrm{TM}} 200$. The relative fluorescence intensity for each value was calculated by normalizing the mean of all technical replicates to the mean of the corresponding $\mathrm{T}$ values. With increasing numbers of DNA strand breaks, the amount of double stranded DNA and thus the resulting fluorescence intensity decreases.

\subsection{Annexin V APC/PI flow cytometry}

For the determination of apoptosis induction, THP-1 cells were stained with Annexin V APC (A35110, Invitrogen) and propidium iodide (PI; P3566, Invitrogen) and analyzed via flow cytometry. Briefly, $1.8 \mathrm{~mL}$ cell suspension $\left(5 \times 10^{5}\right.$ cells $\left./ \mathrm{mL}\right)$ was treated with genotoxins as described in Section 2.2. After the washing steps, cells were resuspended in RPMI medium and incubated for $16 \mathrm{~h}$ at $37^{\circ} \mathrm{C}$ in a humidified atmosphere with $5 \% \mathrm{CO}_{2}$. Cells were harvested $\left(300 \mathrm{~g}, 4^{\circ} \mathrm{C}, 10 \mathrm{~min}\right)$ and resuspended in $500 \mu \mathrm{L} \mathrm{An-}$ nexin binding buffer. For each sample, 2 FACS vials were filled with $100 \mu \mathrm{L}$ cell suspension for one stained and one unstained control sample. One drop of Annexin V APC Ready Flow ${ }^{\mathrm{TM}}$ Reagent and $5 \mu \mathrm{L}$ PI were added to the stained sample. After $15 \mathrm{~min}$ incubation at RT, $400 \mu \mathrm{L}$ Annexin binding buffer was added. Samples were analyzed via BD FACSLyric ${ }^{\mathrm{TM}}$ Research System. Gates were set as illustrated in Figure $\mathrm{S}^{1}$.

\subsection{AlamarBlue ${ }^{T M}$ cell viability assay}

For the analysis of cell viability, $100 \mu \mathrm{L}$ THP-1 cell suspension $\left(5 \times 10^{5}\right.$ cells $\left./ \mathrm{mL}\right)$ was seeded in triplicates for each sample in a 96-well plate and $2 \mu \mathrm{M}$ camptothecin (CPT; CAS: 2114454, Sigma-Aldrich), $200 \mu \mathrm{M} \mathrm{KBrO}_{3}, 20 \mu \mathrm{M} \mathrm{H}_{2} \mathrm{O}_{2}$ or $50 \mu \mathrm{M}$ MMS were added to the cell suspension. Cells were incubated for $16 \mathrm{~h}$ at $37^{\circ} \mathrm{C}$ in a humidified atmosphere with $5 \% \mathrm{CO}_{2} .10 \mu \mathrm{L}$ AlamarBlue $^{\mathrm{TM}}$ cell viability reagent (DAL1025, Invitrogen) was added to each well. After $4 \mathrm{~h}$ of incubation, fluorescence intensity (excitation: $535 \mathrm{~nm}$, emission: $590 \mathrm{~nm}$ ) was measured in a TECAN 


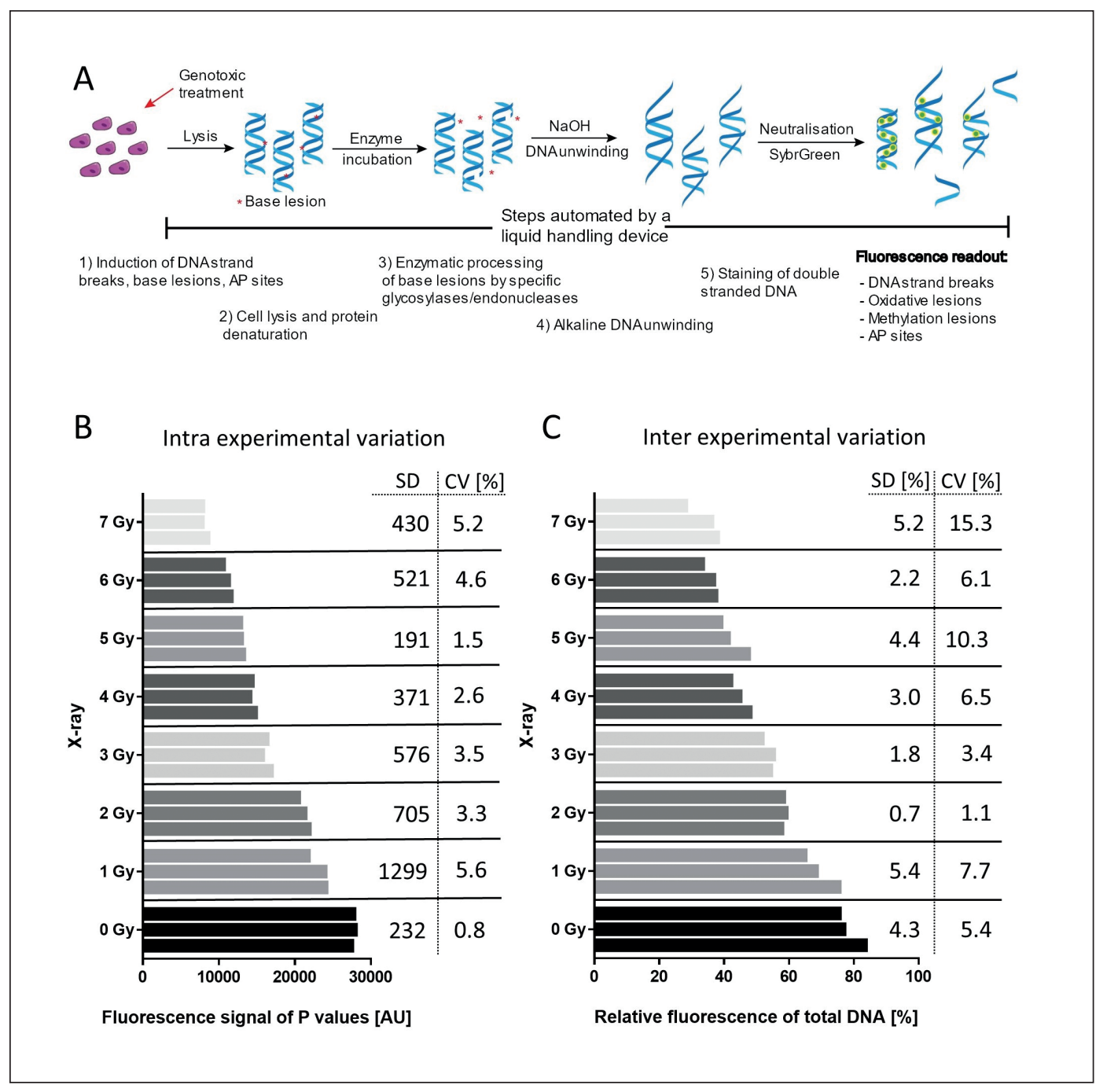

Fig. 1: The enzymemodified FADU assay

(A) Essential steps in the emFADU assay. (B,C) THP-1 cells were irradiated with increasing X-ray doses, and DNA strand breaks were measured via emFADU assay. (B) Intra-experimental variation of 3 technical replicates. Fluorescence signal of $3 \mathrm{P}$ values with the corresponding standard deviation and the coefficient of variation are shown. (C) Interexperimental variation of 3 independent experiments with their corresponding standard deviation and coefficient of variation are shown.

infinite ${ }^{\mathrm{TM}} 200$. Fluorescent intensity of each sample was normalized to the untreated control.

\subsection{Detection of DNA damage in plasmid DNA}

Fpg-sensitive sites and 8-oxodG were determined upon treatment with 3-morpholinosydnonimine (Sin 1, CAS 16142-271, Calbiochem) via emFADU assay and LC-MS/MS in purified plasmid DNA, respectively (Müller et al., 2013). For the detection of methylation lesions upon treatment with MMS via FADU in purified plasmid DNA, $8 \mathrm{U}$ hAAG and $0.5 \mathrm{U}$ APE1 were used instead of Fpg. For determination of 7-methylguanine $(7 \mathrm{mG})$, its ion transition of $166>149$ was detected, as described by $\mathrm{Hu}$ et al. (2012). In parallel to the detection of 8 -oxodG and $7 \mathrm{mG}$, the number of nucleosides was determined and used for normalization. As external standards, 8-oxodG (CAS: 88847-89-6, Sigma-Aldrich), 7mG (CAS: 578-76-7, Sigma-Aldrich), dA (CAS: 16373-93-6, Sigma-Aldrich), dG (CAS: 312693-72-4, Sigma-Aldrich), dT (CAS: 50-89-5, Sigma-Aldrich), and dC (CAS: 951-77-9, Sigma-Aldrich) were used.

\subsection{Statistical analysis}

Statistical analysis was conducted using GraphPad Prism 6 software. For the analysis of experiments with one parameter, a oneway ANOVA followed by Dunnett's multiple comparisons test was performed (Fig. 2A,E; Fig. S1A ${ }^{1}$ ). For the analysis of experiments with two parameters, a two-way ANOVA followed by Sidak's multiple comparisons test was performed (Fig. 2B-D,F; $3 \mathrm{~A}-\mathrm{D})$. $\mathrm{P}$ values are reported by asterisks $\left(* \mathrm{p}<0.05,{ }^{* *} \mathrm{p}<0.01\right.$, $* * * \mathrm{p}<0.001)$.

\section{Results}

\subsection{Detection of DNA strand breaks, oxidative lesions, and methylation lesions in THP-1 cells}

As previously described, the FADU method can be used to detect oxidative DNA lesions in purified plasmid DNA (Müller et al., 2013). We reproduced these findings by measuring the formation of Fpg sensitive sites upon Sin-1 treatment of plasmid DNA (Fig. 

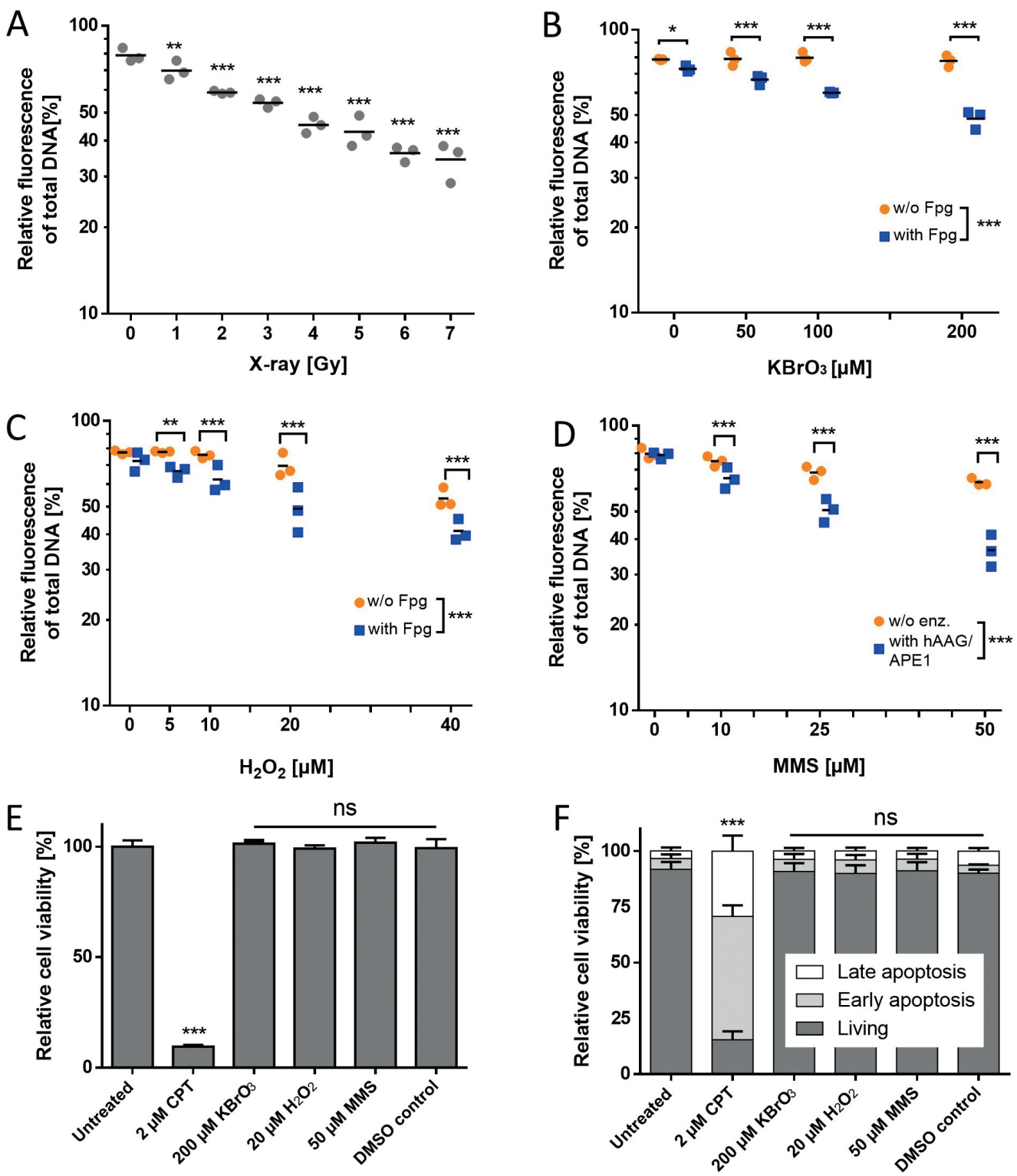

Fig. 2: Detection of DNA strand breaks, oxidative lesions, and methylation lesions in THP-1 cells

(A) THP-1 cells were irradiated on ice with X-ray doses as indicated. DNA SBs were measured via the emFADU assay. Three independent experiments with 3 technical replicates per data point were performed. Statistical analysis: One-way ANOVA with Dunnett's multiple comparisons test ${ }^{* *} \mathrm{p}<0.01$, $\left.{ }^{* *} \mathrm{p}<0.001\right)$. (B,C) THP-1 cells were treated for $2 \mathrm{~h}$ with $\mathrm{KBrO}_{3}$ or for 30 min with $\mathrm{H}_{2} \mathrm{O}_{2}$ as indicated. DNA SBs and Fpg-sensitive sites were measured via the emFADU assay. (D) THP-1 cells were treated for $2 \mathrm{~h}$ with the indicated concentration of MMS. DNA SBs and hAAG/APE1-sensitive sites were measured via the emFADU assay. (B-D) For each data set, 3 independent experiments with 3 technical replicates per data point were performed. Statistical analysis: Two-way ANOVA with Sidak's multiple comparisons test $\left({ }^{* *} \mathrm{p}<0.01,{ }^{* * *} \mathrm{p}<0.001\right)$. (E,F) THP-1 cells were treated with the indicated genotoxins $(\mathrm{CPT}: 16 \mathrm{~h}, \mathrm{KBrO} 3 / \mathrm{MMS}: 2 \mathrm{~h}$, $\mathrm{H}_{2} \mathrm{O}_{2}$ : $30 \mathrm{~min}$ ). (E) $16 \mathrm{~h}$ after treatment, cells were stained with AlamarBlue ${ }^{\mathrm{TM}}$. Fluorescence intensity was measured after $4 \mathrm{~h}$. Three independent experiments with 3 technical replicates per data point were performed. Statistical analysis: One-way ANOVA with Dunnett's multiple comparisons test compared to untreated control ( ${ }^{* *} \mathrm{p}<0.001$, ns: not significant). (F) Cells were stained with Annexin V APC and PI $16 \mathrm{~h}$ after treatment. Fluorescence intensities of individual cells were analyzed via flow cytometry. Three independent experiments were performed. Statistical analysis: Two-way ANOVA with Sidak's multiple comparisons test compared to untreated control $\left({ }^{* * *} p<0.001\right.$; ns, not significant). 
$\left.\mathrm{S} 1 \mathrm{~A} ; \mathrm{S} 2 \mathrm{~A}, \mathrm{~B}^{1}\right)$. We could show that this approach is also suitable for the assessment of chemically induced methylation lesions as well as AP sites (Fig. S1A; S2C,D ${ }^{1}$ ). Figure S1A ${ }^{1}$ shows that the number of hAAG- and APE1 specific lesions increases upon treatment with $10 \mathrm{mM}$ MMS, reflecting the induction of methylation lesions and AP sites, respectively. As indicated, the incubation with hAAG only did not result in the formation of DNA SBs. For verification of the enzyme specificity, we determined the number of 8 -oxodG and $7 \mathrm{mG}$ via LC-MS/MS in parallel to the FADU experiments in plasmid DNA. Treatment of plasmid DNA with increasing concentrations of Sin-1 led to an increase of Fpg-sensitive sites and an increase of 8-oxodG (Fig. S2A,B ${ }^{1}$ ). Treatment of plasmid DNA with increasing concentrations of MMS led to an increase of hAAG/APE1-sensitive sites and $7 \mathrm{mG}$ (Fig. S2C,D ${ }^{1}$ ). Having shown the specificity of Fpg and hAAG for oxidation- and methylation-induced DNA damage, respectively, we then transferred this to a cellular setting.

As described in Section 2.3 and illustrated in Figure 1A, we present here a novel automated screening method for the detection of DNA strand breaks and glycosylase-specific base modifications in human cells. We used the cell line THP-1, which is derived from an acute monocytic leukemia, to establish the method. As the principle of the FADU method relies on the detection of DNA strand interruption-induced DNA unwinding, we first used X-rays to induce DNA SBs. Cells were irradiated on ice and DNA SBs were measured via the emFADU assay protocol. To show the intra-experimental variation of the assay, we analyzed technical triplicates of $\mathrm{P}$ values. As shown in Figure $1 \mathrm{~B}$, the coefficient of variation ranged from 0.8 to $5.6 \%$. To analyze the inter-experimental variation, we conducted three independent experiments. Results in Figure 1C show that the standard deviation of three experiments is below 5.4\% for all X-ray doses. However, the coefficient of variation of the highest dose (7 Gy) was $15.3 \%$, indicating that the induction of heavy DNA damage leads to an increased sample variation between experiments. We also analyzed the inter- and intra-experimental variation of $\mathrm{F}$ and $\mathrm{hA}$ values and found a standard deviation comparable to the $\mathrm{P}$ value (data not shown).

$\mathrm{Next}$, we analyzed the genotoxicity of X-rays, $\mathrm{KBrO}_{3}, \mathrm{H}_{2} \mathrm{O}_{2}$ and MMS in THP-1 cells via the emFADU assay. Increasing $\mathrm{X}$-ray doses led to a decrease of the fluorescence signal, indicating an induction of SBs (Fig. 2A). THP-1 cells were treated for $2 \mathrm{~h}$ with various concentrations of $\mathrm{KBrO}_{3}$ for induction of oxidative lesions. The treatment did not lead to the induction of SBs but to a significant induction of Fpg-sensitive sites already at a concentration of $50 \mu \mathrm{M}$ (Fig. 2B). We could also show that low concentrations of $\mathrm{H}_{2} \mathrm{O}_{2}$ lead to an increase of Fpg-sensitive sites, whereas higher concentrations lead to the formation of SBs without a further increase of Fpg-sensitive sites, which indicates that in this case the upper limit of detection has almost been reached due to saturation (Fig. 2C), i.e., if the DNA is heavily damaged, the unwinding is very fast, and it is not possible to record any additional damage. Further, THP-1 cells were treated with MMS to induce DNA methylation lesions, which could be measured by using a combination of hAAG and APE1. Results in Figure 2D show a slight induction of SBs and a significant increase of hAAG/APE1-sensitive sites upon MMS treatment.

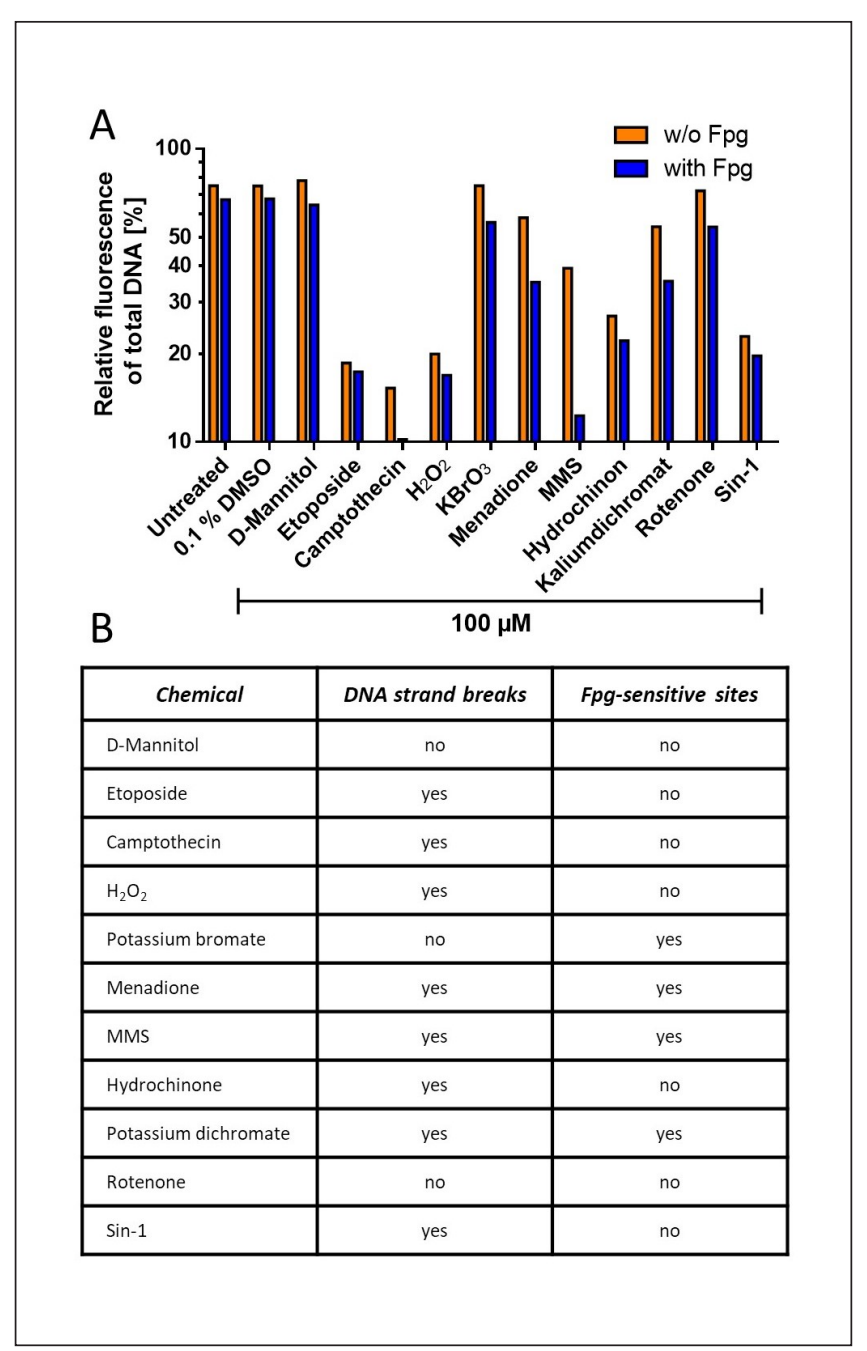

Fig. 3: Genotoxicity screening

(A) $5 \mu \mathrm{L}$ of an anonymized genotoxin solution ( $10 \mathrm{mM}$ ) was added to $500 \mu \mathrm{L}$ THP-1 cell suspension. After $1 \mathrm{~h}$ incubation at $37^{\circ} \mathrm{C}$, DNA SBs and Fpg-sensitive sites were measured via the emFADU assay. For $\mathrm{T}, \mathrm{P}$ and $\mathrm{F}$ value, three technical replicates were used $(n=1)$. (B) Positive induction of DNA SBs was determined via Formula 1; positive induction of Fpg-sensitive sites was determined via Formula 2 (Section 3.2).

To determine whether the applied genotoxin doses are cytotoxic, we measured cell viability via AlamarBlue ${ }^{\mathrm{TM}}$ assay and Annexin V APC/PI flow cytometry $16 \mathrm{~h}$ after treatment. The topoisomerase I inhibitor CPT was used as a positive control. $200 \mu \mathrm{M}$ $\mathrm{KBrO}_{3}, 20 \mu \mathrm{M} \mathrm{H}_{2} \mathrm{O}_{2}$ and $50 \mu \mathrm{M}$ MMS, respectively, did not affect viability of THP-1 cells, whereas incubation with CPT led to a massive reduction of viable cells (Fig. 2E). The applied genotoxic doses showed no induction of apoptosis compared to the significant apoptosis induction upon CPT treatment (Fig. 2F).

Taken together, the data presented in Figure 2 show that the emFADU assay is suitable for the detection of SBs, oxidative and methylation lesions induced by subcytotoxic concentrations of $\mathrm{KBrO}_{3}(200 \mu \mathrm{M}), \mathrm{H}_{2} \mathrm{O}_{2}(20 \mu \mathrm{M})$ and MMS $(50 \mu \mathrm{M})$. 


\subsection{Establishment of a prediction rule for genotoxicity}

To establish a preliminary prediction rule for positive genotoxins, we calculated the mean standard deviation of all data points of all experiments shown in Figure 2 (each was analyzed in three independent experiments). The mean standard deviation of the X-ray experiments was $3.49 \%$ (see Fig. 1C, 2A). The mean standard deviation of all data points (Fig. 2A-D) was 3.52\%, indicating that the addition of enzymes did not increase assay variation substantially. This mean standard deviation $\sigma_{\text {assay }}$ was used to define a result as positive if its relative fluorescence differs at least by three times $\sigma_{2}$ from the untreated control. Therefore, a substance $\mathrm{X}$ is defined as a DNA strand break or Fpg-sensitive site inducing agent if $y(X) \geq 0$.

Formula 1 for DNA strand breaks:

$$
y_{S B}(X)=\left(\frac{P}{T}\right)_{c t r}-\left(\frac{P}{T}\right)_{X}-3 \sigma_{\text {assay }}
$$

Formula 2 for Fpg-sensitive sites:

$$
y_{F p g}(X)=\left(\frac{P}{T}\right)_{X}-\left(\frac{F}{T}\right)_{X}-\left(\frac{P}{T}\right)_{c t r}+\left(\frac{F}{T}\right)_{c t r}-3 \sigma_{a s s a y}
$$

In a first proof-of-principle experiment, 10 known genotoxins were used in a blinded experiment. Additionally, D-mannitol, a known non-genotoxin was included. All substances were employed at a concentration of $100 \mu \mathrm{M}$ in THP-1 cells for 1 hour at $37^{\circ} \mathrm{C}$ in a humidified atmosphere. As shown in Figure 3, 9 of the 10 genotoxins were identified correctly and 4 substances were identified as inducers of Fpg-sensitive sites.

\subsection{Detection of DNA strand breaks, oxidative lesions, and methylation lesions in PBMCs and HeLa cells}

Having shown the functionality of the emFADU assay as a genotoxicity screening method in immortalized suspension cells, we further explored its applicability to PBMCs. PBMCs were isolated from fresh blood of healthy donors. First, we confirmed that the new protocol of the emFADU assay, like the original FADU protocol, is suitable for the detection of X-ray induced SBs (Fig. 4A). Moreover, treatment with $1 \mathrm{mM} \mathrm{KBrO}_{3}$ led to a significant induction of Fpg-sensitive sites (Fig. 4B), whereas treatment with $25 \mu \mathrm{M}$ MMS led to the induction of both Fpg- and hAAG/ APE1-specific sites.

In addition, we extended our approach to the assessment of genotoxicity in adherent cells. To do this, HeLa cells were seeded the day before genotoxic treatment and all assay volumes were reduced by $50 \%$ as the commercial 96 well plates only hold $300 \mu \mathrm{L}$ per well. $\mathrm{H}_{2} \mathrm{O}_{2}$ treatment resulted in a dose-dependent decrease in the fluorescence signal (Fig. 4C). Furthermore, as shown in Figure $4 \mathrm{D}$, treatment with $1 \mathrm{mM} \mathrm{KBrO}_{3}$ and $50 \mu \mathrm{M}$ MMS also led to glycosylase-specific DNA lesions in HeLa cells.

\section{Discussion}

The automated FADU assay was developed as a very sensitive and fast method for the analysis of DNA SBs in human cells. The use of a liquid handling device reduces operator bias and increases sample throughput. Therefore, the automated FADU as- say is an attractive alternative to the commonly used comet assay (Moreno-Villanueva, 2009). Whereas glycosylase-modified versions of the comet assay have already been described, the FADU assay was so far limited to DNA SBs (Collins, 2014). With the enzyme-modified version of the FADU assay (emFADU), we developed a screening method for the parallel assessment of SBs, oxidative lesions, and methylation lesions in human cells. The detection of oxidants and methylating agents could improve the sensitivity of the FADU. Furthermore, this version provides mechanistic information.

First, a cell-free approach was used to describe the functionality and specificity of Fpg, hAAG and APE1. Figure S1 $\mathrm{A}^{1}$ clearly shows that Fpg cleaves Sin-1-induced DNA lesions, whereas hAAG alone is not able to cleave MMS-induced DNA lesions. Therefore, the combination with the endonuclease APE1 is necessary to convert the resulting AP sites into detectable DNA SBs. Moreover, Figure $\mathrm{S}_{1} \mathrm{~A}^{1}$ shows that MMS induces APE1-sensitive sites, indicating the spontaneous depurination of DNA methylation lesions. Attempts were made to measure the spontaneous depurination in cells, too, but no formation of AP sites was detectable in MMS-treated THP-1 cells in first experiments. Therefore, we assume that the depurination of methylation lesions is slower in cellular DNA compared to purified plasmid DNA. We then verified the specificity of Fpg, hAAG and APE1 via the detection of oxidation- and methylation-induced DNA damage in plasmid DNA using the FADU assay together with LC-MS/MS analysis (Fig. S2 ${ }^{1}$ ). We could show that the peroxynitrite generator Sin-1 leads to an increase of Fpg-sensitive sites and 8-oxodG in plasmid DNA. Additionally, MMS treatment led to an increase in hAAG/ APE1-sensitive sites and, in one exemplary experiment, we could show the induction of $7 \mathrm{mG}$ in parallel. We were also able to detect the induction of $7 \mathrm{mG}$ in MMS-treated THP1 cells via LC-MS/ MS (data not shown), but, most probably due to spurious oxidation during DNA purification, it was not possible to detect any induction of 8-oxodG in cellular DNA via mass spectrometry.

For cell-based experiments, we used 96 well plates and triplicates for each of the 4 different values $(\mathrm{T}=$ total $\mathrm{DNA}, \mathrm{P}=\mathrm{SBs}$, $\mathrm{hA}=\mathrm{SBs}+\mathrm{hAAG} / \mathrm{APE} 1$-sensitive sites, $\mathrm{F}=\mathrm{SBs}+$ Fpg-sensitive sites). Although not all parameters were analyzed in every experiment, it was shown in Figure 4 that DNA strand breaks, Fpg-sensitive sites and hAAG/APE1-sensitive sites can be measured in parallel. Thus, it is possible to analyze all parameters of 8 different samples within one plate in a 3-hour run. Depending on the liquid handling system, it is possible to run multiple plates in parallel for high-throughput screening. Moreover, having shown that the intra-experimental variation is quite low (CV $<6 \%$ ), it should be possible to omit technical replicates (Fig. 1B) so that more samples can be measured per plate.

For the detection of Fpg-sensitive sites, we treated cells with $\mathrm{KBrO}_{3}$. It was previously shown that $\mathrm{KBrO}_{3}$ selectively leads to 8-oxodG in cellular DNA in a glutathione (GSH) dependent manner (Murata et al., 2001). We hypothesize that a high level of GSH in THP-1 cells allows for the high sensitivity to $\mathrm{KBrO}_{3}$ (Fig. 2B) compared to PBMCs and HeLa cells, where up to 20 times higher doses were necessary for the induction of Fpg-sensitive sites (Fig. 4B,D). This sensitivity was never described before and could become important in analyzing bromate contami- 


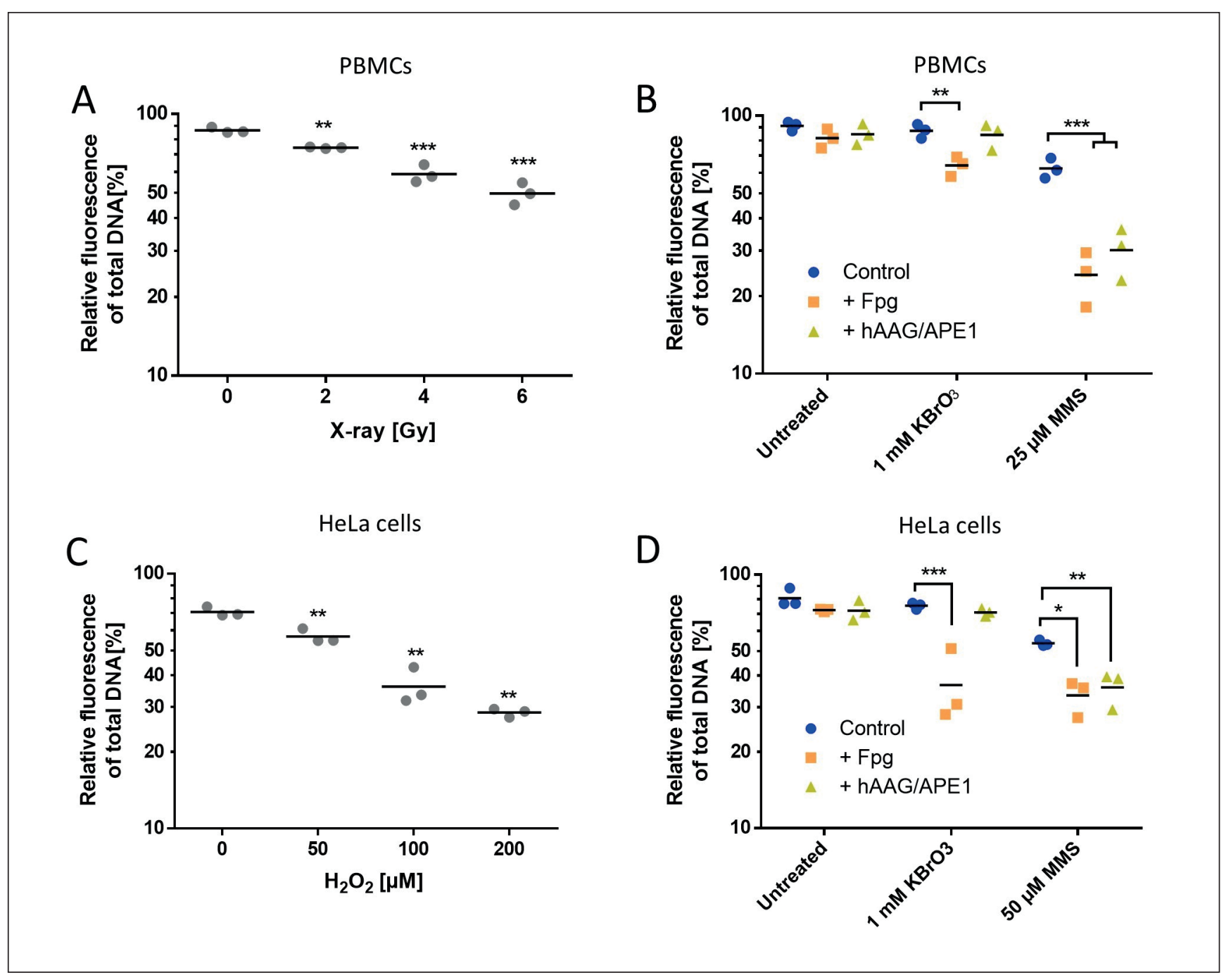

Fig. 4: Detection of DNA strand breaks, oxidative lesions, and methylation lesions in PBMCs and HeLa cells

$(A, B)$ PBMCs were isolated from peripheral blood of healthy human donors. (A) PBMCs were irradiated on ice with $X$-ray doses as indicated. DNA SBs were measured via the emFADU assay. (B) PBMCs were treated with $\mathrm{KBrO}_{3}$ or MMS for $2 \mathrm{~h}$. DNA SBs, Fpg-sensitive sites, and hAAG/APE1-sensitive sites were measured via the emFADU assay. (C) HeLa cells were treated with $\mathrm{H}_{2} \mathrm{O}_{2}$ for 30 min. DNA SBs were measured via the emFADU assay. (D) HeLa cells were treated with $\mathrm{KBrO}_{3}$ or MMS for $2 \mathrm{~h}$. DNA SBs, Fpg-sensitive sites and hAAG/APE1-sensitive sites were measured via the emFADU assay. $(A, C)$ Three independent experiments with 3 technical replicates per data point were performed. Statistical analysis: One-way ANOVA with Dunnett's multiple comparisons test compared to untreated control $\left({ }^{* *} p<0.01,{ }^{* \star *} p<0.001\right)$. (B,D) Three independent experiments with 3 technical replicates per data point were performed. Statistical analysis: Two-way ANOVA with Sidak's multiple comparisons test $\left({ }^{*} \mathrm{p}<0.05,{ }^{* *} \mathrm{p}<0.01,{ }^{* * *} \mathrm{p}<0.001\right)$.

nated samples, which is of great interest, for example in the now more commonly used potentially genotoxic ozonation of water (Aljundi, 2011; Wu et al., 2019). We showed that $\mathrm{KBrO}_{3}$ treatment selectively leads to Fpg-sensitive sites without direct induction of SBs. Therefore, $\mathrm{KBrO}_{3}$ served as a positive control for subsequent experiments.

We used MMS, which was shown to primarily induce 7-methylguanine and 3-methyladenine, to establish the detection of methylation lesions. Within DNA, these bases are efficiently removed by hAAG (Beranek, 1990; Wyatt and Pittman, 2006). Since the monofunctional hAAG does not bind to AP sites, we combined it with APE1, which recognizes and cleaves AP sites, leaving a DNA SB (Abner et al., 2001; Levin and Demple, 1990). While not formally investigated in this study, we assume that our method should be suitable for the assessment of chemically-induced AP sites by the use of APE1 only, but, as described previously, we were not able to detect the formation of AP sites in MMS treated THP-1 cells. In line with the literature (Speit et al., 2004), Figure $4 \mathrm{~B}, \mathrm{D}$ shows that Fpg recognizes both oxidative and methylation lesions. Since human 8-oxoguanine-DNA glycosylase recognizes only oxidative DNA lesions like 8-oxodG and formamidopyrimidine, Fpg could be replaced with this more specific glycosy- 
lase (Sidorenko et al., 2009). However, by measuring Fpg- and hAAG/APE1-sensitive sites in parallel, we can easily distinguish between oxidative and methylation lesions.

At this point, it must be mentioned that any residual chemical that interferes with the fluorescence signal could alter the readout. For example, doxorubicin shows autofluorescence and would lead to an artificial increase in the fluorescence signal. Nevertheless, such interferences are easily detectable by the comparison of $\mathrm{T}$ values of the control sample and chemically treated samples. Additionally, inhibitors of the enzymes used here could lead to false-negative results. To exclude such interference, the enzyme activity could be measured via the use of pre-treated plasmid DNA by the FADU assay.

To prove that the emFADU assay can be used as a screening method for genotoxic substances, we conducted one exemplary experiment with 10 genotoxins and one non-genotoxin. A difference of the value of $10.57 \%$ compared to the control sample, i.e., three times the mean assay standard deviation, was the criterion for definition as a positive hit. This very conservative method identified 9 genotoxins correctly. Although rotenone, a commonly known genotoxin and oxidant, was not detected as a genotoxic substance, potassium bromate was just identified as an oxidizing agent. Furthermore, low concentrations of hydrogen peroxide were shown to induce Fpg-sensitive sites (Fig. 2C), which are not detectable after high-dose exposure (Fig. 3). This shows that exposure time and compound concentration are indeed important issues in genotoxicity testing.

Having shown the proof-of-principle of the emFADU assay, further experiments are planned to assess the sensitivity and specificity of the method. We plan to analyze a large sample set of known genotoxins and non-genotoxins, as well as unclassified substances. In parallel, cytotoxicity studies should be conducted. As shown in Figure 2, due to different reaction mechanisms, the optimal treatment time for different substances varies. Therefore, the best procedure needs to be investigated with a larger sample set. As a next step, the emFADU assay shall be transferred to other laboratories to investigate reproducibility. Here, we presume a high reproducibility due to the use of a liquid handling device.

Due to the high number of chemicals released via drugs, cosmetics, pesticides or food additives, the need for risk assessment testing is undeniable. Different tests for mutagenicity like the Ames test, micronucleus test or chromosomal aberration test have different levels of sensitivity and specificity, leading to false positive and false negative results (Kumar et al., 2018). Here, we provide the basis for a new tool to assess the genotoxic potential of chemicals in a high-throughput format. This could gain importance especially in the early development of compounds and could help to reduce the huge number of animals used in routine toxicity testing. Moreover, besides mutagenicity, the emFADU assay provides useful additional information with regards to specific kinds of DNA damage.

Taken together, based on the automation via a liquid handling system, we have developed a fast and convenient method to assess oxidative and methylation-induced nucleobase lesions in DNA. As described, one run is done in a 96-well plate and takes three hours with costs of less than $10 €$ per 96-well plate for all consumables. In contrast, one CometChip ${ }^{\circledR}$ is more than 20 times more expensive. Additionally, our emFADU assay provides higher throughput with minimal workload. We have also demonstrated its applicability to human PBMCs, thus enabling the use of the emFADU assay in population studies. However, the usefulness of our novel technique to reflect the real-life setting in patient-derived PBMCs needs to be confirmed. Besides cells in suspension, we showed that adherent cells, such as HeLa, can also be used to study genotoxicity. Moreover, with the ability to distinguish between several kinds of DNA damage, the new assay should facilitate the study of DNA repair mechanisms in living cells to address the function of specific proteins involved in repair pathways.

\section{References}

Abner, C. W., Lau, A. Y., Ellenberger et al. (2001). Base excision and DNA binding activities of human alkyladenine DNA glycosylase are sensitive to the base paired with a lesion. J Biol Chem 276, 13379-13387. doi:10.1074/jbc.M010641200

Aljundi, I. H. (2011). Bromate formation during ozonation of drinking water: A response surface methodology study. Desalination 277, 24-28. doi:10.1016/j.desal.2011.03.090

Beken, S., Kasper, P. and van der Laan, J.W. (2016). Regulatory acceptance of alternative methods in the development and approval of pharmaceuticals. Adv Exp Med Biol 856, 33-64. doi:10.1007/978-3-319-33826-2_3

Beranek, D. T. (1990). Distribution of methyl and ethyl adducts following alkylation with monofunctional alkylating agents. Mutat Res 231, 11-30. doi:10.1016/0027-5107(90)90173-2

Bernstein, C., Bernstein, H., Payne, C. M. et al. (2002). DNA repair/pro-apoptotic dual-role proteins in five major DNA repair pathways: Fail-safe protection against carcinogenesis. Mutat Res 511, 145-178. doi:10.1016/S1383-5742(02)00009-1

Birnboim, H. C. and Jevcak, J. J. (1981). Fluorometric method for rapid detection of DNA strand breaks in human white blood cells produced by low doses of radiation. Cancer Res 41, 18891892.

Bouvard, V., Loomis, D., Guyton, K. Z. et al. (2015). Carcinogenicity of consumption of red and processed meat. Lancet Oncol 16, 1599-1600. doi:10.1016/S1470-2045(15) 00444-1

Brunborg, G., Rolstadaas, L. and Gutzkow, K. B. (2018). Electrophoresis in the Comet Assay. In O.-M. Boldura and C. Baltă (eds.), Electrophoresis - Life Sciences Practical Applications. InTech. doi:10.5772/intechopen.76880

Cassano, J. C., Roesslein, M., Kaufmann, R. et al. (2020). A novel approach to increase robustness, precision and highthroughput capacity of single cell gel electrophoresis. ALTEX 37, 95-109. doi:10.14573/altex.1906252

Ciccia, A. and Elledge, S. J. (2010). The DNA damage response: Making it safe to play with knives. Mol Cell 40, 179-204. doi:10.1016/j.molcel.2010.09.019

Collins, A. R. (2014). Measuring oxidative damage to DNA and its repair with the comet assay. Biochim Biophys Acta 1840, 794-800. doi:10.1016/j.bbagen.2013.04.022

Cross, A. J. and Sinha, R. (2004). Meat-related mutagens/carcinogens in the etiology of colorectal cancer. Environ $\mathrm{Mol}$ Mutagen 44, 44-55. doi:10.1002/em.20030 
Enciso, J. M., Gutzkow, K. B., Brunborg, G. et al. (2018). Standardisation of the in vitro comet assay: Influence of lysis time and lysis solution composition on the detection of DNA damage induced by X-rays. Mutagenesis 33, 25-30. doi:10. 1093/mutage/gex039

Ersson, C., Møller, P., Forchhammer, L. et al. (2013). An ECVAG inter-laboratory validation study of the comet assay: Inter-laboratory and intra-laboratory variations of DNA strand breaks and FPG-sensitive sites in human mononuclear cells. Mutagenesis 28, 279-286. doi:10.1093/mutage/get001

Hartwig, A., Dally, H. and Schlepegrell, R. (1996). Sensitive analysis of oxidative DNA damage in mammalian cells: Use of the bacterial Fpg protein in combination with alkaline unwinding. Toxicol Lett 88, 85-90. doi:10.1016/0378-4274(96)03722-8

Hoeijmakers, J. H. J. (2009). DNA damage, aging, and cancer. N Engl J Med 361, 1475-1485. doi:10.1056/NEJMra0804615

Hu, C.-W., Chen, C.-M., Ho, H. H. et al. (2012). Simultaneous quantification of methylated purines in DNA by isotope dilution LC-MS/MS coupled with automated solid-phase extraction. Anal Bioanal Chem 402, 1199-1208. doi:10.1007/ s00216-011-5559-1

Knudsen, L. E., Smith, A., Törnqvist, E. et al. (2019). Nordic symposium on "toxicology and pharmacology without animal experiments - Will it be possible in the next 10 years?" Basic Clin Pharmacol Toxicol 124, 560-567. doi:10.1111/bcpt.13193

Kumar, A., Dobrovolsky, V., Dhawan A. et al. (2018). Mutagenicity: Assays and Applications. Elsevier. ISBN: 9780128092521

Levin, J. D. and Demple, B. (1990). Analysis of class II (hydrolytic) and class I (beta-lyase) apurinic/apyrimidinic endonucleases with a synthetic DNA substrate. Nucleic Acids Res 18, 5069-5075. doi:10.1093/nar/18.17.5069

Lindahl, T. (1993). Instability and decay of the primary structure of DNA. Nature 362, 709-715. doi:10.1038/362709a0

Moreno-Villanueva, M., Pfeiffer, R., Sindlinger, T. et al. (2009). A modified and automated version of the 'fluorimetric detection of alkaline DNA unwinding' method to quantify formation and repair of DNA strand breaks. BMC Biotechnol 9, 39. doi: 10.1186/1472-6750-9-39

Moreno-Villanueva, M., Eltze, T., Dressler, D. et al. (2011). The automated FADU-assay, a potential high-throughput in vitro method for early screening of clastogenicity. ALTEX 28, 295303. doi:10.14573/altex.2011.4.295

Müller, N., Moreno-Villanueva, M., Fischbach, A. et al. (2013). An automated Fpg-based FADU method for the detection of oxidative DNA lesions and screening of antioxidants. Toxicology 310, 15-21. doi:10.1016/j.tox.2013.05.006

Murata, M., Bansho, Y., Inoue, S. et al. (2001). Requirement of glutathione and cysteine in guanine-specific oxidation of DNA by carcinogenic potassium bromate. Chem Res Toxicol 14, 678-685. doi:10.1021/tx000209q

O'Connor, T. R. and Laval, J. (1989). Physical association of the 2,6-diamino-4-hydroxy-5N-formamidopyrimidine-DNA glycosylase of Escherichia coli and an activity nicking DNA at apurinic/apyrimidinic sites. Proc Natl Acad Sci U S A 86, 5222-5226. doi:10.1073/pnas.86.14.5222

Pflaum, M., Will, O. and Epe, B. (1997). Determination of steady-state levels of oxidative DNA base modifications in mammalian cells by means of repair endonucleases. Carcinogenesis 18, 2225-2231. doi:10.1093/carcin/18.11.2225

Sidorenko, V. S., Grollman, A. P., Jaruga, P. et al. (2009). Substrate specificity and excision kinetics of natural polymorphic variants and phosphomimetic mutants of human 8-oxoguanine-DNA glycosylase. FEBS J 276, 5149-5162. doi:10.1111/ j.1742-4658.2009.07212.x

Speit, G., Schütz, P., Bonzheim, I. et al. (2004). Sensitivity of the FPG protein towards alkylation damage in the comet assay. Toxicol Lett 146, 151-158. doi:10.1016/j.toxlet.2003.09.010

Sykora, P., Witt, K., Revanna, R. et al. (2018). Next generation high throughput DNA damage detection platform for genotoxic compound screening. Sci Rep 8, 2771. doi:10.1038/s41598018-20995-w

Tchou, J., Kasai, H., Shibutani, S. et al. (1991). 8-oxoguanine (8-hydroxyguanine) DNA glycosylase and its substrate specificity. Proc Natl Acad Sci U S A 88, 4690-4694. doi:10.1073/ pnas.88.11.4690

Wu, Q.-Y., Zhou, Y.-T., Li, W. et al. (2019). Underestimated risk from ozonation of wastewater containing bromide: Both organic byproducts and bromate contributed to the toxicity increase. Water Res 162, 43-52. doi:10.1016/j.watres.2019.06.054

Wyatt, M. D. and Pittman, D. L. (2006). Methylating agents and DNA repair responses: Methylated bases and sources of strand breaks. Chem Res Toxicol 19, 1580-1594. doi:10.1021/ tx060164e

\section{Conflict of interest}

The authors declare that they have no conflict of interest.

\section{Acknowledgements}

This study was supported by the Baden-Württemberg Ministry for Science, Research and Art (MWK Baden-Württemberg) through the "Kooperatives Promotionskolleg (KPK) InViTe Sigmaringen/Konstanz", by funding MM as a doctoral student. We would like to thank Dr María Moreno-Villanueva for her advice on the automated FADU assay protocol and Mr Thilo Sindlinger for technical support. We thank Prof. Marcus Groettrup, Dr Annette Sommershof and Ms Ricarda Schwab from the University of Konstanz Flow-Cytometry Platform for their advice and support concerning flow cytometric analyses. We thank Prof. Jörg Bergemann, University of Applied Sciences, Albstadt-Sigmaringen, Germany for his input as a member of the KPK InViTe thesis advisory committee for MM. We thank Prof. Aswin Mangerich for critical reading of the manuscript. 